\title{
A Hemispheric Division of Labor Aids Mental Rotation
}

\author{
Kazuhito Yoshizaki \\ Aichi Shukutoku University
}

\author{
Daniel H. Weissman \\ University of Michigan
}

\author{
Marie T. Banich \\ University of Colorado at Boulder
}

\begin{abstract}
In the present study, we investigated whether a hemispheric division of labor is most advantageous to performance when lateralized inputs place unequal resource demands on the left and right cerebral hemispheres. In each trial, participants decided whether 2 rotated letters, presented either in the same visual field (within-field trials) or in opposite visual fields (across-field trials), were both of normal orientation, or whether one was normal and the other was mirror-reversed. To discriminate a letter's orientation, one must rotate the letter to the upright position. Therefore, we manipulated whether the two letters imposed similar or dissimilar demands on cognitive resources by varying the number of degrees that each letter needed to be rotated to reach the upright position. As predicted, in 2 experiments we found that the across-field advantage increased as the number of degrees each letter needed to be rotated became more dissimilar. These findings support a current model of hemispheric interactions, which posits that an unequal hemispheric distribution of cognitive load allows the cerebral hemispheres to take the lead for different aspects of cognitive processing.
\end{abstract}

Although it is widely recognized that the left and right cerebral hemispheres process information quite differently (Hellige, 1993; Springer \& Deutsch, 1998), relatively little is known about how interactions between the cerebral hemispheres modulate cognitive processing. Recently, Banich and colleagues (Banich, 1998; Banich \& Belger, 1990) have proposed that a division of processing across the hemispheres increases the processing capacity of the brain. According to Banich (1998), a division of critical information across the hemispheres allows for a more efficient hemispheric division of labor than does directing critical information to a single hemisphere. In support of this view, Banich and colleagues have consistently demonstrated that as the resource demands for a task increase, across-field processing (i.e., a division of critical information across the left and right visual fields) becomes more advantageous to performance relative to withinfield processing (e.g., Banich \& Belger, 1990; Belger \& Banich, 1992; Passarotti, Banich, Sood, \& Wang, 2002; Weissman \& Banich, 1999, 2000), a result that has also been reported by other groups (Compton, 2002; Coney, 1985; Koivisto, 2000; Norman, Jeeves, Milne, \& Ludwig, 1992; Yoshizaki, 2000; Yoshizaki \& Tsuji, 2000; Zhang \& Feng, 1999). Converging evidence to support this model comes from neuroimaging studies, which demon-

Kazuhito Yoshizaki, Department of Psychology and Communication, Aichi Shukutoku University, Aichi, Japan; Daniel H. Weissman, Department of Psychology, University of Michigan; Marie T. Banich, Institute of Cognitive Science and Department of Psychology, University of Colorado at Boulder.

This research was supported in part by a grant from Grand-in-Aid for Scientific Research (No. 15530480) to Kazuhito Yoshizaki. We are grateful to Kimiko Kato, Mayumi Deguchi, and Kumiko Kawamoto for conducting the present experiments.

Correspondence concerning this article should be addressed to Kazuhito Yoshizaki, Department of Psychology and Communication, Aichi Shukutoku University, 9, Katahira-Nagakute, Nagakute-cho, Aichi 480-1197, Japan. E-mail: yoshizak@asu.aasa.ac.jp strate that bilateral activity (which could be taken to indicate a hemispheric division of labor) often increases as task demands become greater (e.g., Jonides et al., 1997; Klingberg, O'Sullivan, \& Roland, 1997; Pollman, Zaidel, \& von Cramon, 2003; Tsukiura et al., 2002).

According to Banich (1998), the across-field advantage is determined jointly by (a) the resource demands imposed by a task and (b) the time costs associated with interhemispheric communication via the corpus callosum. When a task is relatively complex, the benefits afforded by additional processing resources outweigh the time costs associated with interhemispheric communication, leading to an across-field advantage. On the other hand, when a task is relatively simple, the need for additional processing resources does not outweigh the time costs associated with interhemispheric communication, leading to a within-field advantage.

Of course, it is difficult to predict, a priori, whether across-field processing will facilitate the performance of a given task relative to within-field processing. For this reason, we have used our model to make predictions about the relative size of the across-field advantage for two or more tasks that vary in their complexity, rather than about the presence or absence of an across-field advantage for a single task. Our model predicts that across-field processing should be relatively more advantageous to performance for a more complex task than for a simpler task, regardless of whether either task is associated with a within-field or an acrossfield advantage. In support of this hypothesis, a large within-field advantage for a relatively less complex task can shift to a smaller within-field advantage for a relatively more complex task (e.g., Weissman, Banich, \& Puente, 2000) or to no difference between within-field and across-field processing (e.g., Passarotti et al., 2002, Experiment 1), rather than to an across-field advantage. In other cases, a small across-field advantage for a less complex task can become a larger one for a more complex task (e.g., Belger \& Banich, 1992). These findings support our model by showing that across-field processing becomes relatively more advantageous to performance as task complexity increases, regardless of whether 
any particular task exhibits a within-field or an across-field advantage. Moreover, the findings illustrate that the effects of acrossfield processing on performance change gradually as task complexity varies, rather than in an all-or-none fashion.

In the present study, we investigated whether the size of the across-field advantage also depends on the extent to which task inputs impose unequal cognitive demands on the two cerebral hemispheres. This hypothesis was motivated by Banich and Belger's (1990) claim that an unequal hemispheric division of inputs helps to produce the across-field advantage for complex tasks. Specifically, these authors suggested that when the processing load on the hemispheres is unequal, it creates conditions favorable to each cerebral hemisphere taking the lead for different cognitive operations underlying performance. For example, they argued that on across-field trials in three-item letter-matching tasks, the hemisphere that receives two letters takes the lead for perceptual processing while the hemisphere that receives only one letter takes the lead for decision making, a hypothesis that was supported in a subsequent study (Banich, Stolar, Heller, \& Goldman, 1992). Thus, a key prediction of Banich and colleagues' model is that the across-field advantage for complex tasks should be greater when task inputs impose unequal cognitive demands on the cerebral hemispheres than when they impose equal demands.

In line with this view, Weissman et al. (2000) found that the size of the across-field advantage is greater when the inputs to each hemisphere are unequal (e.g., one item in the left visual field and two in the right visual field). In this study, the authors used the same letter-matching tasks as were used by Banich and Belger (1990). In the physical identity task, participants decided whether a letter beneath fixation (e.g., "A") was perceptually identical to either of two letters above fixation (e.g., "A" and "B"), whereas in the name identity task, the participants decided whether a letter beneath fixation (e.g., "a") had the same name as either of two letters above fixation (e.g., "A" and "B"). For both tasks, Weissman et al. (2000) manipulated whether the hemispheres received unequal processing loads or equal processing loads by varying whether there was just one letter beneath fixation (three-item trials) or two letters, one in each visual field (four-item trials). In support of the view that an unequal hemispheric division of labor facilitates across-field processing, Weissman et al. (2000) found that the across-field advantage was significantly larger for three-item than for four-item displays in both the physical identity and the naming identity tasks. Moreover, this effect occurred even though mean reaction time was significantly longer for the four-item than for the three-item tasks, demonstrating that the manner in which information is divided between the hemispheres, and not task difficulty, is the primary determinant of the across-field advantage.

Although Weissman et al.'s (2000) findings support Banich and colleagues' (Banich, 1998; Banich \& Belger, 1990) model, they leave uncertain whether equating the number of inputs directed to each hemisphere dilutes the advantage of across-field processing by equating (a) the perceptual load directed to each hemisphere and/or (b) the cognitive load imposed on each hemisphere. In the present study, we directly investigated whether equating the cognitive load imposed on the left and right cerebral hemispheres reduces the size of the across-field advantage.

To investigate this hypothesis, we used a mental rotation task, as prior data indicate that the cognitive load imposed by mental rotation is directly related to the number of degrees a stimulus must be rotated. For example, Cooper and Shepard (1973) found that the time needed to decide whether a letter is in the normal orientation or mirror-reversed is directly proportional to the number of degrees the letter has been rotated away from the upright position. This finding led these authors to argue that participants needed to rotate the letter to the upright position before they could decide whether it was normally oriented or mirror-reversed. Such effects have also been observed in mental rotation matching tasks, in which two rotated letters are presented and participants must decide whether both are in the normal orientation or whether one is normally oriented and the other is mirror-reversed (Fischer \& Pellegrino, 1988; Francis \& Irwin, 1997; Hishitani, 1983). Thus, we reasoned that a mental rotation matching task was ideally suited for testing our hypothesis.

Of importance, our mental rotation matching task allowed us to manipulate the cognitive load imposed on each hemisphere while holding constant the perceptual load that was directed to each hemisphere. In each trial of our task (Figure 1), we presented a pair of rotated letters, either in the same visual-field (within-field trials) or in opposite visual fields (across-field trials). Participants were asked to decide whether both letters were normally oriented or whether one was normally oriented and the other was mirrorreversed. We also presented two task-irrelevant stimuli (i.e., two circles) on each trial, so that the perceptual load directed to each hemisphere (i.e., two stimuli) was equated in within-field and across-field trials. To manipulate the cognitive load imposed on each hemisphere, we varied whether the two letters needed to be rotated by similar or dissimilar numbers of degrees in order to reach the upright position. In two experiments, we predicted the across-field advantage would be maximal under conditions in which unequal mental rotation demands (i.e., cognitive loads) were imposed on the left and right cerebral hemispheres.

\section{Experiment 1}

In Experiment 1, we varied the number of degrees each letter needed be rotated in three pairings: 5 degrees and 85 degrees (5-85), 25 degrees and 65 degrees (25-65), and 45 degrees and 45

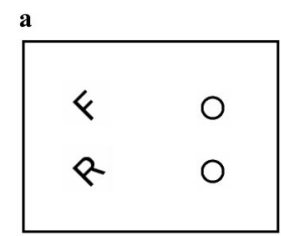

c

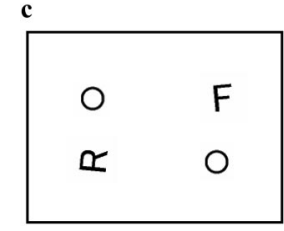

b

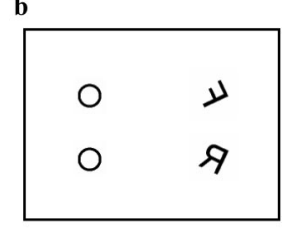

d

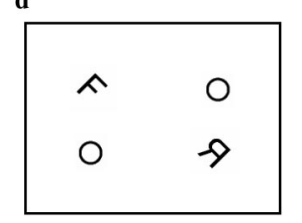

Figure 1. Examples of stimulus configurations used in Experiment 1. Shown are sample (a) within-left visual field (LVF) match trials (the 45-45 degree condition), (b) within-right visual field (RVF) mismatch trials (the 25-65 degree condition), (c) across-bottom LVF match trials (the 5-85 degree condition), and (d) across-bottom RVF mismatch trials (the 45-45 degree condition). 
degrees (45-45), while holding constant the total number of degrees the two letters needed to be rotated in order to reach the upright position (i.e., 90 degrees). This design allowed us to investigate how varying the cognitive load to each hemisphere affected the size of the across-field advantage while holding constant the total demands on mental rotation processes. We predicted that the across-field advantage would grow larger as the difference in cognitive load imposed on the two hemispheres increased.

\section{Method}

\section{Participants}

Twenty-four right-handed undergraduate students (12 women and 12 men: mean age $=20.8$ years) participated in this experiment. They had normal or corrected-to-normal vision. Their handedness was evaluated by the HN Handedness Inventory (Hatta \& Nakatsuka, 1975). Participants received course credit for their participation.

\section{Stimuli}

Target letter stimuli were the letters " $F$ " and "R", presented in MS UI Gothic font. Each letter was displayed in four different angular orientations, ranging from $5^{\circ}$ to $85^{\circ}$ clockwise and counterclockwise from the normal upright in $20^{\circ}$ steps. Each letter target was displayed in each orientation condition in both a normal and backward (mirror-image) version. Letters subtended $1.4^{\circ}$ by $1.0^{\circ}$ of visual angle. The distractor stimulus was a circle "O," which subtended $0.9^{\circ}$ by $0.9^{\circ}$ of visual angle. All stimuli were displayed in black on a white background.

On every trial, two letters and two distractors were simultaneously presented on the screen. Each target and distractor was located either $3.0^{\circ}$ to the left or to the right of the central fixation point, and at a visual angle of $3.0^{\circ}$ above or below the level of the central fixation point. As Figure 1 shows, there were four types of match trials: within-left visual field (within LVF), within-right visual field (within RVF), across-bottom LVF (across b-LVF) and across-bottom RVF (across b-RVF). In addition, there were an equal number of mismatch trials. In half of these, the bottom item was presented in the LVF, and in half it was presented in the RVF.

Each target letter pair consisted of the letter "F," which was displayed in the upper visual field, and the letter "R," which was displayed in the lower visual field. Each letter was rotated in the same direction (clockwise or counterclockwise) from the upright position. The two letters were rotated by a total of 90 degrees, but the number of degrees each letter was rotated varied systematically across three rotation conditions. The degrees of rotation for the three conditions were $5^{\circ}$ and $85^{\circ}, 25^{\circ}$ and $65^{\circ}$, and $45^{\circ}$ and $45^{\circ}$.

In match trials, both letters were oriented normally. In mismatch trials, one letter was oriented normally while the other was mirrorreversed.

\section{Apparatus}

An IBM-compatible personal computer equipped with a 17-in. extended graphics array (XGA) color monitor (Model CPD-E230, Sony, Tokyo, Japan) was used to present the stimuli. Trial presentation and the recording of response choices and latencies were controlled by SuperLab Pro for Windows Version 2.04 software
(Cedrus Company, San Pedro, CA). Participants' responses were recorded by a Microsoft-compatible serial mouse.

\section{Experimental Design}

There were three within-subject variables: type of judgment (match, mismatch), rotation condition $(5-85,25-65,45-45)$, and presentation mode (within-field, across-field).

\section{Procedure}

The participants were tested individually. They were strongly encouraged to maintain central fixation at all times during the task. The viewing distance from participants' eyes to the monitor was $37 \mathrm{~cm}$ and was maintained with a chin-rest.

The sequence of events in each trial was as follows. First, there was a warning tone and a 1,000-ms central fixation point. Second, the two letters and two distractors were simultaneously presented for $180 \mathrm{~ms}$. The participants were required to judge, as quickly and as accurately as possible, whether both target letters were in the normal orientation (match trials) or whether one was in the normal orientation while the other was mirror-reversed (mismatch trials) through a button press made with the index or middle finger. All reaction times in the present study were measured from stimulus onset to response. Half of the participants responded with the right hand, and the other half responded with the left. The inter-trial interval was 2,000 ms. Nine blocks of 48 trials were presented. The first block was discarded from the analysis because it served as a practice run.

For each participant, the mean of the individual reaction times in each experimental condition was calculated for correct responses. Trials with response times less than $200 \mathrm{~ms}$, or exceeding 1,500 $\mathrm{ms}$, were treated as errors. Such outliers comprised less than $0.8 \%$ of all trials. Reaction time analyses in the present study were carried out only for correct responses in match trials. Mismatch trials were omitted from the analyses because, in these trials, participants might simply rotate one of the two letters to the upright position and, if it happened to be the letter in mirrorreverse orientation, immediately reach a mismatch decision. Thus, while correct performance in match trials required that both letters be rotated to the upright position, correct performance in mismatch trials did not. Because our hypotheses about within-field and across-field processing could only be tested by assuming that both letters were rotated to the upright position, we omitted mismatch trials from the analyses. However, mismatch trials were important to include in the experimental design because they forced participants to rotate each letter to the upright position in match trials.

\section{Results}

\section{Reaction Time}

An analysis of variance (ANOVA) with two within-subject variables-rotation condition $(5-85,25-65,45-45)$ and presentation mode (within-field, across-field)—was carried out using mean response times for correct match judgments. Table 1 shows mean reaction times and their standard deviations for correct responses in each experimental condition. Table 2 shows mean error rates and their standard deviations in each experimental condition. 
Table 1

Mean Reaction Times (in Milliseconds) and Standard Deviations for Correct Responses in Experiment 1

\begin{tabular}{|c|c|c|c|c|c|c|c|c|c|c|c|c|}
\hline \multirow[b]{3}{*}{ Condition } & \multicolumn{4}{|c|}{ 45-45 condition } & \multicolumn{4}{|c|}{ 25-65 condition } & \multicolumn{4}{|c|}{ 5-85 condition } \\
\hline & \multicolumn{3}{|c|}{ Within } & \multirow[b]{2}{*}{ Across } & \multicolumn{3}{|c|}{ Within } & \multirow[b]{2}{*}{ Across } & \multicolumn{3}{|c|}{ Within } & \multirow[b]{2}{*}{ Across } \\
\hline & LVF & RVF & $\begin{array}{l}\text { Weighted } \\
\text { average }\end{array}$ & & LVF & RVF & $\begin{array}{l}\text { Weighted } \\
\text { average }\end{array}$ & & LVF & RVF & $\begin{array}{l}\text { Weighted } \\
\text { average }\end{array}$ & \\
\hline \multicolumn{13}{|l|}{ Match } \\
\hline$M$ & 503 & 521 & 511 & 502 & 553 & 551 & 552 & 522 & 656 & 649 & 653 & 593 \\
\hline$S D$ & 103 & 116 & 106 & 66 & 108 & 115 & 107 & 99 & 124 & 119 & 117 & 107 \\
\hline \multicolumn{13}{|l|}{ Mismatch } \\
\hline$M$ & 558 & 566 & 562 & 571 & 573 & 573 & 573 & 572 & 617 & 638 & 628 & 613 \\
\hline$S D$ & 103 & 110 & 105 & 97 & 122 & 110 & 113 & 102 & 141 & 143 & 139 & 113 \\
\hline
\end{tabular}

Note. The numbers in the three conditions refer to the number of degrees each letter needed be rotated: 45 degrees and 45 degrees (45-45), 25 degrees and 65 degrees (25-65), and 5 degrees and 85 degrees (5-85), while holding constant the total number of degrees the two letters needed to be rotated in order to reach the upright position (i.e., 90 degrees). In match trials, both letters were oriented normally. In mismatch trials, one letter was oriented normally while the other was mirror-reversed. LVF = left visual field; RVF = right visual field.

There was a significant main effect of rotation condition, $F(2$, 46) $=155.67, p<.01$, partial eta squared $\left(\eta_{\text {partial }}^{2}\right)=.87$. Tukey honestly significant difference (HSD) tests $(\alpha=.05)$ showed that mean response time in the $45-45$ condition (507 ms) was significantly faster than in the 25-65 (537 ms) and in the 5-85 condition (623 ms). Furthermore, mean response time was significantly faster in the 25-65 condition than in the 5-85 condition. There was also a significant main effect of presentation mode, $F(1$, 23) $=29.07, p<.01, \eta_{\mathrm{p}}^{2}=.56$, indicating that across-field processing $(539 \mathrm{~ms})$ was significantly faster than within-field processing (572 $\mathrm{ms})$.

As illustrated in Figure 2, there was also a significant interaction between rotation condition and presentation mode, $F(2$, 46) $=10.44, p<.01, \eta_{\mathrm{p}}^{2}=.31$. Analyses of simple effects showed that an across-field advantage appeared in both the 25-65 condition, $F(1,69)=11.77, p<.01$, and the 5-85 condition, $F(1$, $69)=45.05, p<.01$. However, there was no significant difference between the within- and across-field conditions in the 45-45 condition, $F(1,69)=1.01, p=.32$. Most important, as hypothesized, Tukey HSD tests $(\alpha=.05)$ indicated that the across-field advantage was significantly larger in the $5-85$ condition $(60 \mathrm{~ms})$ than in the other two conditions ( $9 \mathrm{~ms}$ in the $45-45$ condition, 31 $\mathrm{ms}$ in the 25-65 condition) and that the across-field advantage was significantly larger in the 25-65 condition than in the 45-45 condition.

\section{Error Rates}

An analogous ANOVA carried out with mean error rates yielded results that were consistent with the ANOVA on mean reaction times reported above. First, there was a significant main effect of rotation condition, $F(2,46)=16.66, p<.01, \eta_{\mathrm{p}}^{2}=.42$. Though there was no difference in mean error rates between the $45-45$ (.027) and the 25-65 conditions (.036), the mean error rate for the 5-85 condition (.072) was significantly higher than in the other two conditions (Tukey HSD test, $\alpha=.05$ ). Second, there was a significant main effect of presentation mode, $F(1,23)=24.64$, $p<.01, \eta_{\mathrm{p}}^{2}=.52$. Error rates in the across-field condition (.030) were significantly lower than in the within-field condition (.060). The interaction between rotation condition and presentation mode did not reach conventional levels of significance, $F(2,46)=2.73$, $p=.08, \eta_{\mathrm{p}}^{2}=.10$, but, similar to the analyses of RT presented

Table 2

Mean Error Rates and Standard Deviations in Experiment 1

\begin{tabular}{|c|c|c|c|c|c|c|c|c|c|c|c|c|}
\hline \multirow[b]{3}{*}{ Condition } & \multicolumn{4}{|c|}{ 45-45 condition } & \multicolumn{4}{|c|}{ 25-65 condition } & \multicolumn{4}{|c|}{ 5-85 condition } \\
\hline & \multicolumn{3}{|c|}{ Within } & \multirow[b]{2}{*}{ Across } & \multicolumn{3}{|c|}{ Within } & \multirow[b]{2}{*}{ Across } & \multicolumn{3}{|c|}{ Within } & \multirow[b]{2}{*}{ Across } \\
\hline & LVF & RVF & $\begin{array}{c}\text { Weighted } \\
\text { average }\end{array}$ & & LVF & RVF & $\begin{array}{l}\text { Weighted } \\
\text { average }\end{array}$ & & LVF & RVF & $\begin{array}{l}\text { Weighted } \\
\text { average }\end{array}$ & \\
\hline \multicolumn{13}{|l|}{ Match } \\
\hline$M$ & .023 & .042 & .033 & .022 & .039 & .060 & .049 & .022 & .086 & .107 & .096 & .047 \\
\hline$S D$ & .040 & .071 & .042 & .032 & .054 & .056 & .038 & .025 & .086 & .115 & .070 & .047 \\
\hline \multicolumn{13}{|l|}{ Mismatch } \\
\hline$M$ & .021 & .049 & .035 & .052 & .070 & .068 & .069 & .070 & .104 & .089 & .096 & .108 \\
\hline$S D$ & .039 & .081 & .043 & .060 & .061 & .100 & .063 & .061 & .067 & .062 & .053 & .076 \\
\hline
\end{tabular}

Note. The numbers in the three conditions refer to the number of degrees each letter needed be rotated: 45 degrees and 45 degrees (45-45), 25 degrees and 65 degrees (25-65), and 5 degrees and 85 degrees (5-85), while holding constant the total number of degrees the two letters needed to be rotated in order to reach the upright position (i.e., 90 degrees). In match trials, both letters were oriented normally. In mismatch trials, one letter was oriented normally while the other was mirror-reversed. $\mathrm{LVF}=$ left visual field; $\mathrm{RVF}=$ right visual field. 


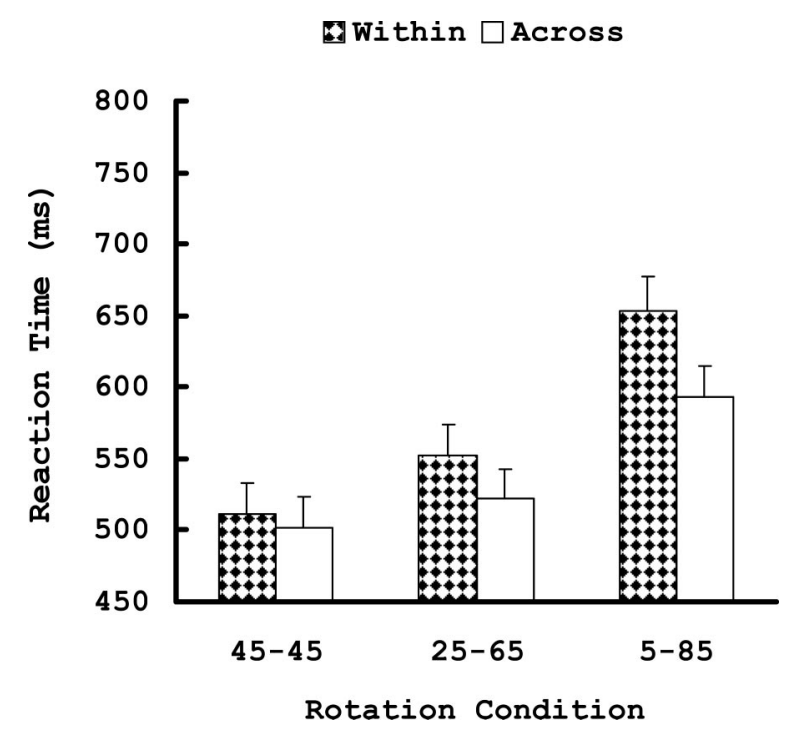

Figure 2. Mean reaction times for correct match decisions in Experiment 1 as a function of the presentation mode (within vs. across visual field) and letter-rotation condition. Error bars indicate standard error.

earlier, tests of simple effects revealed a significant across-field advantage in the $25-65, F(1,69)=5.80, p<.05$, and $5-85$ conditions, $F(1,69)=19.00, p<.01$, but not in the $45-45$ condition, $F(1,69)=.84, p=.36$. Furthermore, as predicted, the across-field advantage (Tukey HSD: $\alpha=.05$ tests) was significantly larger in the 5-85 condition (.049) than in the 45-45 condition (.011). However, the across-field advantage in the 25-65 condition (.026) did not differ significantly from the across-field advantages in the other two rotation conditions.

\section{Discussion}

In Experiment 1, we varied the number of degrees that each letter needed to be rotated in order to reach the upright position while holding constant, at 90 degrees, the total number of degrees of rotation that were required in each trial. As predicted, the across-field advantage increased as the number of degrees each letter needed to be rotated became more and more unequal. This result appears to support our hypothesis that the across-field advantage derives, at least in part, from the ability to direct unequal cognitive loads to the left and right cerebral hemispheres.

\section{Experiment 2}

In Experiment 2, we investigated a possible alternative explanation for our findings in Experiment 1 . Specifically, we investigated whether the size of the across-field advantage might have been driven by the cognitive demands imposed by the letter that was rotated farthest away from the upright position. For example, the relatively large cognitive demands imposed by a letter that needed to be rotated 85 degrees might have led to our observing the largest across-field advantage in the 5-85 rotation condition. Clearly, this alternative view differs from our hypothesis that the size of the across-field advantage in Experiment 1 was driven by the difference in the number of degrees that each letter needed to be rotated in order to reach the upright position.

To distinguish between these competing hypotheses in Experiment 2, we held constant the number of degrees that the more severely rotated letter in each trial needed to be rotated in order to reach the upright position (i.e., 85 degrees) while varying, across three conditions, the number of degrees that the other letter needed to be rotated $(5,40$, or 75 degrees). We reasoned that if the across-field advantage in Experiment 1 was driven by the cognitive demands imposed by the letter that was rotated farthest away from the upright position, then the size of the across-field advantage in Experiment 2 should be invariant across the three rotation conditions above. On the other hand, if the across-field advantage in Experiment 1 was driven by the ability to direct unequal cognitive loads to the two hemispheres, then the across-field advantage in Experiment 2 should be largest in the 5-85 condition and smaller in the 40-80 and 75-85 conditions.

\section{Method}

\section{Participants}

Twenty-four right-handed undergraduate students (12 women and 12 men, mean age $=20.9$ years old) participated in the present experiment. They were recruited and screened as in Experiment 1, but none had participated in Experiment 1.

\section{Apparatus}

The apparatus was identical to that in Experiment 1.

\section{Stimuli}

The stimuli were prepared in the same manner as in Experiment 1 , except that each letter was displayed in four different angular orientations: $5^{\circ}, 40^{\circ}, 75^{\circ}$, and $85^{\circ}$.

As in Experiment 1, all of the target letter pairs consisted of a letter "F," which was displayed in the upper visual field, and a letter "R," which was displayed in the lower visual field. Each letter of the pair was rotated in the same direction (clockwise or counterclockwise) from the normal position. There were three rotation conditions: $5^{\circ}$ and $85^{\circ}, 40^{\circ}$ and $85^{\circ}$, and $75^{\circ}$ and $85^{\circ}$. Note that, in each of these conditions, one letter always needed to be rotated by $85^{\circ}$ to reach the upright position. As in Experiment 1 , for match trials, both letters were oriented normally. For mismatch judgment trials, however, one letter was oriented normally while the other was mirror-reversed. Figure 3 shows examples of the stimulus configurations used in Experiment 2.

\section{Experimental Design}

As in Experiment 1, there were three within-subject variables: type of judgment (match, mismatch), rotation condition (5-85, 40-85, 75-85), and presentation mode (within-field, across-field).

\section{Procedure}

The procedure was identical to that of Experiment 1. As in Experiment 1, trials with reaction times less than $200 \mathrm{~ms}$, or 


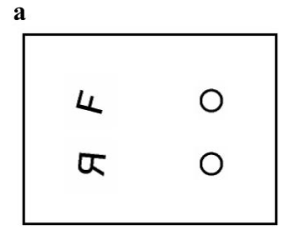

c

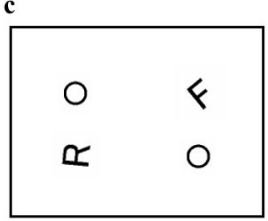

b

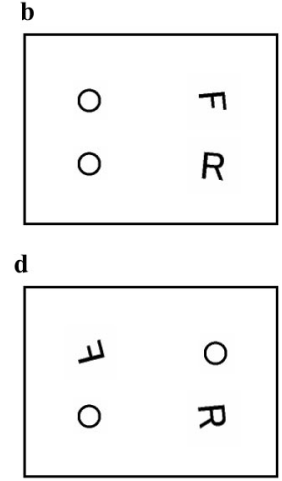

Figure 3. Examples of stimulus configurations used in Experiment 2. Shown are sample (a) within-LVF mismatch trials (the 75-85 degree condition), (b) within-RVF match trials (the 5-85 degree condition), (c) across-bottom LVF match trials (the 40-85 degree condition), and (d) across-bottom RVF mismatch trials (the 75-85 degree condition). $\mathrm{LVF}=$ left visual field; $\mathrm{RVF}=$ right visual field.

exceeding 1,500 ms, were treated as errors. Such outliers comprised less than $0.3 \%$ of all trials in Experiment 2.

\section{Results}

\section{Reaction Time}

An ANOVA with two within-subjects factors-rotation condition (5-85, 40-85, and 75-85) and presentation mode (withinfield, across-field) — was carried out using mean reaction times for correct responses in match trials. Table 3 shows mean reaction times and their standard deviations for correct responses in each experimental condition. Table 4 shows mean error rates and their standard deviations in each experimental condition.

There was a significant main effect of rotation condition, $F(2$, $46)=28.54, p<.01, \eta_{\mathrm{p}}^{2}=.55$. Multiple comparisons via Tukey's HSD test $(\alpha=.05)$ showed that mean reaction time was significantly longer in the 5-85 condition (700 ms) than in the other two conditions (40-85 condition, RT: $652 \mathrm{~ms} ; 75-85$ condition, RT:
$644 \mathrm{~ms}$ ) but that there was no difference in mean reaction time between the $40-85$ and the $75-85$ conditions. There was also a significant main effect of presentation mode, $F(1,23)=68.47$, $p<.01, \eta_{\mathrm{p}}^{2}=.75$, as mean reaction time was significantly faster in across-field trials $(646 \mathrm{~ms})$ than in within-field trials $(684 \mathrm{~ms})$.

Most important, as illustrated in Figure 4, there was a significant interaction between rotation condition and presentation mode, $F(2$, $46)=28.36, p<.01, \eta_{\mathrm{p}}^{2}=.45$. There were significant across-field advantages for both the $5-85, F(1,69)=117.97, p<.01$, and the $40-85$ conditions, $F(1,69)=6.02, p<.05$, but not for the $75-85$ condition, $F(2,46)=.80, p=.38$. As predicted, Tukey HSD tests ( $\alpha=.05)$ revealed that the across-field advantage in the 5-85 condition $(87 \mathrm{~ms}$ ) was significantly larger than that in the other two conditions (20 ms in the $40-85$ condition, $7 \mathrm{~ms}$ in the $75-85$ condition). The across-field advantage in the 40-85 condition, however, did not differ significantly from that in the 75-85 condition. Nonetheless, the results for the 5-85 condition support our view that the ability to direct unequal cognitive load to the two cerebral hemispheres is a powerful determinant of the across-field advantage.

\section{Error Rates}

An analogous ANOVA using mean error rates from match judgment trials yielded effects that were consistent with those observed in the reaction time data. First, there was a marginally significant main effect of rotation condition, $F(2,46)=3.11, p=$ $.05, \eta_{\mathrm{p}}^{2}=.12$. Multiple comparisons via Tukey's HSD tests $(\alpha=$ .05 ) showed that the mean error rate for the 5-85 condition (.059) was significantly greater than that for the 40-85 condition (.040) but did not significantly differ from that in the 75-80 condition (.050). Furthermore, mean error rates did not significantly differ for the 40-85 and the 75-85 conditions (Tukey HSD test, $\alpha=$ $.05)$. There was also a significant main effect of presentation mode, $F(1,23)=20.17, p<.01, \eta_{\mathrm{p}}^{2}=.47$, because error rates were lower on across-field trials (.029) than on within-field trials (.071).

Finally, the interaction between rotation condition and presentation mode approached significance, $F(2,46)=2.82, p=.07$, $\eta_{\mathrm{p}}^{2}=.11$. As observed for the reaction time data, there was a significant across-field advantage for both the 5-85, $F(1$,

Table 3

Mean Reaction Times (in Milliseconds) and Standard Deviations for Correct Responses in Experiment 2

\begin{tabular}{|c|c|c|c|c|c|c|c|c|c|c|c|c|}
\hline \multirow[b]{3}{*}{ Condition } & \multicolumn{4}{|c|}{$75-85$ condition } & \multicolumn{4}{|c|}{$40-85$ condition } & \multicolumn{4}{|c|}{ 5-85 condition } \\
\hline & \multicolumn{3}{|c|}{ Within } & \multirow[b]{2}{*}{ Across } & \multicolumn{3}{|c|}{ Within } & \multirow[b]{2}{*}{ Across } & \multicolumn{3}{|c|}{ Within } & \multirow[b]{2}{*}{ Across } \\
\hline & LVF & RVF & $\begin{array}{l}\text { Weighted } \\
\text { average }\end{array}$ & & LVF & RVF & $\begin{array}{l}\text { Weighted } \\
\text { average }\end{array}$ & & LVF & RVF & $\begin{array}{l}\text { Weighted } \\
\text { average }\end{array}$ & \\
\hline \multicolumn{13}{|l|}{ Match } \\
\hline$M$ & 656 & 639 & 648 & 641 & 664 & 659 & 662 & 642 & 730 & 757 & 743 & 656 \\
\hline$S D$ & 72 & 70 & 65 & 58 & 71 & 67 & 71 & 72 & 87 & 121 & 98 & 69 \\
\hline \multicolumn{13}{|l|}{ Mismatch } \\
\hline$M$ & 679 & 685 & 682 & 704 & 684 & 687 & 686 & 688 & 714 & 696 & 705 & 710 \\
\hline$S D$ & 100 & 96 & 94 & 101 & 85 & 98 & 87 & 86 & 113 & 105 & 105 & 98 \\
\hline
\end{tabular}

Note. The numbers in the three conditions refer to the number of degrees each letter needed to be rotated: 75 degrees and 85 degrees ( $75-85$ ), 40 degrees and 85 degrees (40-85), and 5 degrees and 85 degrees (5-85). Note that one letter always needed to be rotated by 85 degrees in order to reach the upright position. In match trials, both letters were oriented normally. In mismatch trials, one letter was oriented normally while the other was mirror-reversed. $\mathrm{LVF}=$ left visual field; $\mathrm{RVF}=$ right visual field. 
Table 4

Mean Error Rates and Standard Deviations in Experiment 2

\begin{tabular}{|c|c|c|c|c|c|c|c|c|c|c|c|c|}
\hline \multirow[b]{3}{*}{ Condition } & \multicolumn{4}{|c|}{$75-85$ condition } & \multicolumn{4}{|c|}{$40-85$ condition } & \multicolumn{4}{|c|}{ 5-85 condition } \\
\hline & \multicolumn{3}{|c|}{ Within } & \multirow[b]{2}{*}{ Across } & \multicolumn{3}{|c|}{ Within } & \multirow[b]{2}{*}{ Across } & \multicolumn{3}{|c|}{ Within } & \multirow[b]{2}{*}{ Across } \\
\hline & LVF & RVF & $\begin{array}{l}\text { Weighted } \\
\text { average }\end{array}$ & & LVF & RVF & $\begin{array}{l}\text { Weighted } \\
\text { average }\end{array}$ & & LVF & RVF & $\begin{array}{l}\text { Weighted } \\
\text { average }\end{array}$ & \\
\hline \multicolumn{13}{|l|}{ Match } \\
\hline$M$ & .052 & .073 & .063 & .039 & .044 & .076 & .060 & .020 & .070 & .112 & .091 & .028 \\
\hline$S D$ & .067 & .080 & .056 & .039 & .049 & .099 & .060 & .025 & .085 & .088 & .080 & .036 \\
\hline \multicolumn{13}{|l|}{ Mismatch } \\
\hline$M$ & .081 & .047 & .064 & .064 & .073 & .070 & .071 & .083 & .073 & .044 & .058 & .091 \\
\hline$S D$ & .069 & .037 & .042 & .054 & .074 & .075 & .057 & .062 & .067 & .049 & .048 & .072 \\
\hline
\end{tabular}

Note. The numbers in the three conditions refer to the number of degrees each letter needed be rotated: 75 degrees and 85 degrees (75-85), 40 degrees and 85 degrees (40-85), and 5 degrees and 85 degrees (5-85). Note that one letter always needed to be rotated by 85 degrees in order to reach the upright position. In match trials, both letters were oriented normally. In mismatch trials, one letter was oriented normally while the other was mirror-reversed. $\mathrm{LVF}=$ left visual field; $\mathrm{RVF}=$ right visual field.

$69)=21.90, p<.01$, and the $40-85$ conditions, $F(1,69)=8.55$, $p<.01$, but not for the 75-85 condition, $F(1,69)=3.08, p=.08$. As predicted, Tukey HSD tests $(\alpha=.05)$ showed that the acrossfield advantage in the 5-85 condition (.063) was significantly larger than that in the 75-85 condition (.024). The across-field advantage in the 5-85 condition, however, did not differ from that in the 40-85 condition (.040). Furthermore, there was no difference in the size of the across-field advantage for the 40-75 and the $75-85$ conditions.

\section{Discussion}

Experiment 2 was designed to determine whether the acrossfield advantage in Experiment 1 was driven by (a) the cognitive load placed on a single hemisphere or (b) the difference between the cognitive loads imposed on the left and right hemispheres. Our

国Within $\square$ Across

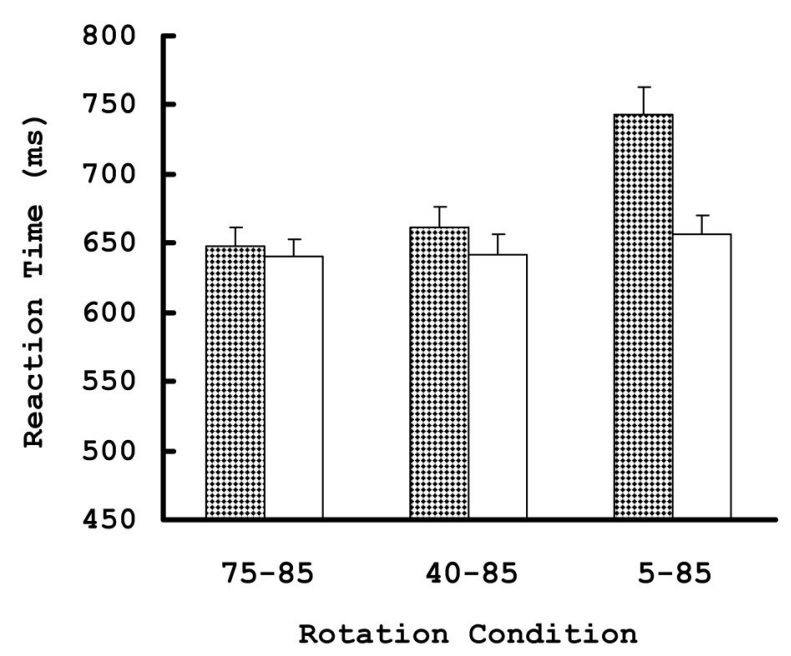

Figure 4. Mean reaction times for correct match decisions in Experiment 2 as a function of the presentation mode (within vs. across visual field) and letter rotation condition. Error bars indicate standard error. findings in Experiment 2 clearly indicate that the across-field advantage is driven by the difference in the cognitive load that is imposed on the two cerebral hemispheres. Given that this result replicates our main finding from Experiment 1, the different participants that took part in Experiments 1 and Experiment 2 appear to have used similar task strategies while performing our mental rotation tasks. Thus, the present findings appear to be quite robust.

\section{General Discussion}

Previous studies have demonstrated that across-field processing becomes more advantageous to performance, relative to withinfield processing, as task complexity increases (Banich, 1998; Banich \& Belger, 1990; Compton, 2002; Koivisto, 2000; Norman et al., 1992; Weissman \& Banich, 1999; Yoshizaki \& Tsuji, 2000; Zhang \& Feng, 1999). Banich and colleagues have posited that the across-field advantage for complex tasks occurs because a hemispheric division of inputs allows the two sides of the brain to take the lead for different processes underlying task performance (e.g., perceptual processing vs. decision-making). Moreover, they have argued that an unequal division of inputs across the two hemispheres facilitates this hemispheric division of cognitive operations (Banich et al., 1992; Weissman et al., 2000).

In the present study, we investigated a key prediction of Banich and colleagues' model, which is that the across-field advantage should be greater when task stimuli impose unequal versus equal cognitive loads on each cerebral hemisphere. To do so, we used a mental rotation matching task in which we could independently vary the number of degrees that each of two letters needed to be rotated in order to reach the upright position. Our main assumption, bolstered by previous research (Cooper \& Shepard, 1973; Fischer \& Pellegrino, 1988; Francis \& Irwin, 1997; Hishitani, 1983), was that the cognitive load imposed by each letter was proportional to the number of degrees that the letter needed to be rotated in order to reach the upright position. As predicted, in two experiments we found that the across-field advantage became greater as the cognitive load imposed on each hemisphere became more unequal.

Experiment 1 demonstrated a powerful effect of directing equal versus unequal cognitive loads to the two hemispheres while controlling for overall cognitive load, as defined by overall de- 
mands on mental rotation processes. Specifically, the across-field advantage increased as the difference in the number of degrees each letter needed to be rotated became greater. However, the total cognitive load on mental rotation processes was held constant at 90 degrees in all trials. Thus, the difference in demands imposed on hemispheric mental rotation processes, rather than overall demands on such processes, appeared to account for our findings.

Experiment 2 tested whether the cognitive load imposed on the more burdened hemisphere in Experiment 1, which was confounded with the difference in cognitive load imposed on the two hemispheres, might account for our findings in Experiment 1. To remove this confound in Experiment 2, every trial included one letter that was rotated 85 degrees from upright and a second letter that was rotated 75,40 , or 5 degrees from upright. We therefore manipulated the similarity of the cognitive load imposed on each hemisphere while holding constant the demands on the more burdened hemisphere (i.e., 85 degrees). In line with our hypothesis and with the findings from Experiment 1, we found that the across-field advantage increased as the difference in cognitive load imposed on the two hemispheres became greater. Thus, independent of overall cognitive load (Experiment 1) and the cognitive load imposed on the more burdened hemisphere (Experiment 2), the across-field advantage became greater when unequal (versus equal) cognitive loads were imposed on the cerebral hemispheres.

Our present findings concur with those of Weissman et al. (2000) as both indicate that the across-field advantage is reduced by equating the cognitive load imposed on each hemisphere. However, in Weissman et al.'s (2000) study, cognitive load and perceptual load were confounded. Thus, the present findings more specifically demonstrate that equating the cognitive load imposed on the two hemispheres reduces the across-field advantage. Interestingly, this reduction occurred in both studies even though imposing equal (versus unequal) cognitive loads on each hemisphere raised overall measures of cognitive load. In Experiment 2 of the present study, equating the cognitive load imposed on each hemisphere increased the total number of degrees the two letters needed to be rotated. In the Weissman et al. (2000) study, equating the cognitive load to each hemisphere involved presenting an extra letter in the display, which needed to be perceptually identified and compared with several other letters in order to reach a match/ mismatch decision. Raising overall demands on cognitive processes usually increases the across-field advantage (Banich \& Belger, 1990; Belger \& Banich, 1992, 1998; Koivisto, 2000; Norman et al., 1992; Weissman \& Banich, 1999). Thus, our finding that equating the cognitive load imposed on each hemisphere reduces the across-field advantage, even when it increases overall cognitive load, demonstrates that the ability to direct unequal cognitive loads to the two hemispheres is a powerful determinant of the across-field advantage.

Of course, given the complexities associated with this area of research, it is important to consider carefully the possible limitations of the present study. As we mentioned earlier, one potential limitation is that the different participants who took part in Experiments 1 and 2 may have engaged different task strategies. However, our finding that the size of the across-field advantage increased with the angular disparity of the two letters in both experiments provided evidence against this possibility. In the paragraphs that follow, we consider several additional possible limitations of the present study that should be considered while interpreting our findings.

Our conclusions regarding the across-field advantage rest upon the assumption that each letter in match trials was rotated to the upright position, as predicted by the existent literature on mental rotation. The present findings support this assumption by suggesting that the two letters in each trial were rotated in parallel. Because distinguishing parallel from serial processing can be difficult (e.g., Townsend \& Wenger, 2004), we now provide a detailed explanation for why the data from the present experiments support a parallel rotation model.

In Experiment 1, our finding that reaction time increased in proceeding from the $45-45$ to the $25-65$ to the 5-85 condition (on both within-field and across-field trials) is clearly inconsistent with the view that the two letters in each trial were serially rotated to the upright position. Indeed, the total number of degrees the two letters needed to be rotated remained constant across these three conditions. However, the gradual increase in reaction time across these three conditions is consistent with parallel rotation of the two letters in each trial because the number of degrees the more severely rotated letter needs to be rotated is increasing across these conditions (i.e., from 45 to 65 to 85). Assuming that both letters are being rotated at the same rate, the time needed to complete a parallel rotation should be determined by the letter that needs to be rotated by the largest number of degrees.

Experiment 2 provides further evidence for parallel rotation of the two letters in each trial. Specifically, the constant reaction times for the different rotation conditions on across-field trials are highly consistent with parallel rotation of the two letters, as the completion time for a parallel rotation in all three conditions would likely be driven by the letter that needs to be rotated 85 degrees. On within-field trials, reaction time increased in proceeding from the $75-85$ to the $40-85$ to the 5-85 condition, which is quite the opposite of what a serial rotation model would predict because the total number of degrees of rotation is decreasing across these three conditions. Thus, both Experiments 1 and 2 rule out a serial rotation model in favor of a parallel rotation model. Furthermore, both experiments indicate that the efficiency of mental rotation processes is lower on within-field than on across-field trials. Next, we review the likely sources for this reduced efficiency in within-field trials.

Prior research suggests that the reduced efficiency of mental rotation on within-field trials may stem from at least two possible sources. First, it may stem from a reduced ability to divide distinct mental rotation processes across the somewhat independent pools of resources that are provided by each cerebral hemisphere, a division of labor that has been posited to become increasingly advantageous as task complexity increases (e.g., Banich \& Belger, 1990; Weissman \& Banich, 1999). Second, it may stem from an inability to divide conflicting processes between the cerebral hemispheres (e.g., Liederman, 1986; Merola \& Liederman, 1985, 1990; Sohn, Liederman, \& Reinitz, 1996). In the present experiments, rotating two letters by different numbers of degrees might have induced conflict. For example, such conflict could have arisen from the need to implement two highly similar, yet slightly distinct, mental rotation processes or from having to continue to rotate one letter after rotation of the other letter was already completed. Given the two possible sources of reduced efficiency on within-field trials that we have reviewed, future studies should further examine the degree to which it is a separation of 
distinct operations across the hemispheres or the separation of conflicting operations across the hemispheres that leads to the across-field advantage for complex tasks.

Thus far, we have emphasized our view that equating the cognitive load directed to each cerebral hemisphere reduces the across-field advantage by making it more difficult for each side of the brain to take the lead for a different aspect of processing (Banich et al., 1992; Weissman et al., 2000). It is important to consider, however, whether hemispheric asymmetries for different aspects of mental rotation might provide a better explanation for the present findings than does our load theory. Mental rotation tasks involve several task components (e.g., stimulus encoding, image generation, maintaining images in a visual buffer, actual rotation, comparison/decision processes, response execution, etc.), and some of these components may be performed better by one hemisphere than by the other (e.g., Harris \& Miniussi, 2003; Hugdahl, Thomsen, \& Ersland, 2006; Zacks, Rypma, Gabrieli, Tversky, \& Glover, 1999). One might therefore ask whether hemispheric asymmetries for various components of our mental rotation tasks could have interacted with the angular disparity of the to-be-rotated letters in each trial to produce the interhemispheric effects that we observed.

Although it is difficult to completely rule out an alternative account of our results that is based on hemispheric asymmetries for different aspects of mental rotation, several arguments weigh heavily against this view. First, the size of the across-field advantage appears to be largely independent of the nature and/or degree of hemispheric asymmetries that are observed for a task (e.g., Compton \& Weissman, 2002; Lopez, Kosson, Weissman, \& Banich, 2007; Weissman \& Banich, 1999, 2000), except in rare cases when a highly lateralized task can only be performed by one side of the brain (Belger \& Banich, 1998). Using the present data as an example, in both experiments the largest across-field advantage was observed in the 5-85 condition, but only in Experiment 2 was the 5-85 condition associated with the largest difference between within-LVF and within-RVF performance. Second, some task manipulations affect performance when the hemispheres interact, but not when either hemisphere works in relative isolation (e.g., Banich \& Karol, 1992). Such findings indicate that qualitatively different processes may be at play when hemispheric interactions underlie performance compared with when a single hemisphere does. Third, when the cerebral hemispheres cooperate to perform a task, they sometimes both adopt the processing mode of the less efficient hemisphere, possibly because it is more difficult for the less efficient hemisphere to adopt the processing mode of the more efficient hemisphere (e.g., Hellige, Taylor, \& Eng, 1989). Thus, when the hemispheres interact, each may underlie operations that it is not specialized to perform. Fourth, a rotation-specific account fails to explain why, even when load is defined completely differently than in the present study (i.e., as the number of inputs rather than as the number of degrees each input needs to be rotated), it is still the case that directing equal versus unequal loads to the two visual fields reduces the efficiency of across-field processing (Weissman et al., 2000). It is, in fact, for this reason that we invoke the general concept of load to account for our findings (rather than a variety of task-specific processes), similar to other researchers who have observed effects of manipulating processing demands that generalize across multiple tasks (e.g., Bavelier, Deruelle, \& Proksch, 2000; Kim, Kim, \& Chun, 2005; Lavie, Hirst, de Fockert, \& Viding, 2004). Given these considerations, it seems highly unlikely that the interhemispheric effects we observed in the present study were driven by hemispheric asymmetries for different aspects of mental rotation.

More broadly, our findings suggesting that two letters may be rotated in parallel, particularly on across-field trials in Experiment 2, add to a growing, yet equivocal, literature on whether mental rotation can be performed in parallel with other cognitive operations. For example, using a dual-task procedure, Van Selst and Jolicoeur (1994) obtained evidence that mental rotation can proceed in parallel with a second task. In contrast, using a similar procedure Ruthruff, Miller, and Lachmann (1995) found only sparse evidence to support the parallel processing view. Adding to this controversy, Heil, Wahl, and Herbst (1999) demonstrated that certain mental rotation effects under dual task conditions are not easily explained by any current theory of mental rotation. These discrepant findings may stem, at least in part, from the fact that processing can be performed either serially or in parallel in dualtask paradigms, depending on the strategy participants are encouraged to adopt (Meyer \& Kieras, 1997a, 1997b). In the present study, we obtained evidence that two letters can be rotated in parallel using a same-different matching task, which lacks many of the complexities that are present in a dual-task situation. Additional studies should be conducted to further elucidate the effects we have reported and to determine more precisely the conditions under which it is possible to rotate two letters in parallel.

Future studies might also investigate whether the interhemispheric effects that we have reported vary with the particular participant population under investigation. The degree to which across-field processing is advantageous for a particular task varies with intellectual giftedness (Singh \& O'Boyle, 2004), and the extent to which across-field processing becomes more advantageous to performance as task complexity increases varies with handedness (e.g., Eviatar, Hellige, \& Zaidel, 1997) and age (Banich, Passarotti, \& Janes, 2000; Reuter-Lorenz, Stanczak, \& Miller, 1999). Thus, individual differences might also modulate the effects of directing unequal versus equal cognitive load to the two cerebral hemispheres on the size of the across-field advantage.

The present findings are also relevant to understanding changes in behavior that are associated with impaired communication between the cerebral hemispheres. Reduced communication via the corpus callosum (i.e., the band of fibers that connects corresponding cortical regions in the left and right hemispheres) can facilitate the performance of certain demanding tasks. For instance, split-brain patients, in whom the corpus callosum has been severed, are better than neurologically intact controls at searching each visual field in parallel for a target stimulus (Luck, Hillyard, Mangun, \& Gazzaniga, 1989). For most demanding tasks, however, impaired communication across the corpus callosum leads to performance decrements. For example, dual-task performance is frequently impaired in split-brain patients relative to controls (Kreuter, Kinsbourne, \& Trevarthen, 1972). More broadly, performance deficits for demanding tasks have been reported for a wide array of clinical syndromes that are associated with callosal injury, such as closed-head injury, attention-deficit disorder, and multiple sclerosis (for a detailed review of these findings, see Banich, 1998). The present results may therefore inform our understanding of these clinical syndromes by suggesting that the deficits exhibited by patients with damage to the corpus callosum may be most pronounced when a task makes unequal demands on the two cerebral hemispheres. 
In sum, we have demonstrated that an unequal division of cognitive load between the cerebral hemispheres facilitates the performance of a complex mental rotation task. Future studies investigating precisely which aspects of cognitive processing are divided between the cerebral hemispheres and whether such a hemispheric division of labor facilitates parallel processing may help to further reveal the source(s) of the across-field advantage.

\section{References}

Banich, M. T. (1998). The missing link: The role of interhemispheric interaction in attentional processing. Brain and Cognition, 36, 128-157.

Banich, M. T., \& Belger, A. (1990). Interhemispheric interaction: How do the hemispheres divide and conquer a task? Cortex, 26, 77-94.

Banich, M. T., \& Karol, D. L. (1992). The sum of the parts does not equal the whole: Evidence from bihemispheric processing. Journal of Experimental Psychology: Human Perception and Performance, 18, 763-784.

Banich, M. T., Passarotti, A. M., \& Janes (2000). Interhemispheric interaction during childhood. I. Neurologically-intact children. Developmental Neuropsychology, 18, 33-51.

Banich, M. T., Stolar, N., Heller, W., \& Goldman, R. (1992). A deficit in right-hemisphere performance after induction of a depressed mood. Neuropsychiatry, Neuropsychology, \& Behavioral Neurology, 5, 20-27.

Bavelier, D., Deruelle, C., \& Proksch, J. (2000). Positive and negative compatibility effects. Perception \& Psychophysics, 62, 100-112.

Belger, A., \& Banich, M. T. (1992). Interhemispheric interaction affected by computational complexity. Neuropsychologia, 30, 923-929.

Belger, A., \& Banich, M. T. (1998). Costs and benefits of integrating information between the cerebral hemispheres: A computational perspective. Neuropsychology, 12, 380-398.

Compton, R. J. (2002). Inter-hemispheric interaction facilitates face processing. Neuropsychologia, 40, 2409-2419.

Compton, R. J., \& Weissman, D. H. (2002). Hemispheric asymmetries in global-local perception: Effects of individual differences in neuroticism. Laterality, 7, 333-350.

Coney, J. (1985). Dual hemispheric processing in a letter matching task. Perception \& Psychophysics, 38, 331-342.

Cooper, L. A., \& Shepard, R. N. (1973). Chronometric studies of the rotation of mental images. In W. G. Chase (Ed.), Visual information processing (pp. 75-176). New York: Academic Press.

Eviatar, Z., Hellige, J. B., \& Zaidel, E. (1997). Individual differences in lateralization: Effects of gender and handedness. Neuropsychology, 11, 562-576.

Fischer, S. C., \& Pellegrino, J. W. (1988). Hemispheric differences for components of mental rotation. Brain and Cognition, 7, 1-15.

Francis, M. A., \& Irwin, R. J. (1997). Cerebral asymmetry and decision strategies in mental rotation: A psychophysical analysis. European Journal of Cognitive Psychology, 9, 225-240.

Harris, I. M., \& Miniussi, C. (2003). Parietal lobe contribution to mental rotation demonstrated with rTMS. Journal of Cognitive Neuroscience, 15, 315-323.

Hatta, T., \& Nakatsuka, Z. (1975). H. N. Handedness Inventory. In S. Ohno (Ed.), Papers for celebration of the 63rd birthday of Prof. Ohnishi (pp. 224-247). Osaka, Japan: Osaka City University Press.

Heil, M., Wahl, K., \& Herbst, M. (1999). Mental rotation, memory scanning, and the central bottleneck. Psychological Research, 62, 48-61.

Hellige, J. B. (1993). Hemispheric asymmetry. Cambridge, MA: Harvard University Press.

Hellige, J. B., Taylor, A. K., \& Eng, T. L. (1989). Interhemispheric interaction when both hemispheres have access to the same stimulus information. Journal of Experimental Psychology: Human Perception and Performance, 15, 711-722.
Hishitani, S. (1983). An experimental study on the parallel operation of image: A case of mental rotation. The Japanese Journal of Psychology, 53, 337-343.

Hugdahl, K., Thomsen, T., \& Ersland, L. (2006). Sex differences in visuo-spatial processing: An fMRI study of mental rotation. Neuropsychologia, 44, 1575-1583.

Jonides, J., Schumacher, E. H., Smith, E. E., Lauber, E. J., Awh, E., Minoshima, S., et al. (1997). Verbal working memory load affects regional brain activation as measured by PET. Journal of Cognitive Neuroscience, 9, 462-475.

Kim, S., Kim, M., \& Chun, M. M. (2005). Concurrent working memory load can reduce distraction. Proceedings of the National Academy of Sciences, 102, 16524-16529.

Klingberg, T., O’Sullivan, B. T., \& Roland, P. E. (1997). Bilateral activation of fronto-parietal networks by incrementing demand in a working memory task. Cerebral Cortex, 7, 465-471.

Koivisto, M. (2000). Interhemispheric interaction in semantic categorization of pictures. Cognitive Brain Research, 9, 45-51.

Kreuter, C., Kinsbourne, M., \& Trevarthen, C. (1972). Are deconnected cerebral hemispheres independent channels? A preliminary study of the effect of unilateral loading on bilateral finger tapping. Neuropsychologia, 10, 453-461.

Lavie, N., Hirst, A., de Fockert, J. W., \& Viding, E. (2004). Load theory of selective attention and cognitive control. Journal of Experimental Psychology: General, 133, 339-354.

Liederman, J. (1986). Subtraction in addition to addition: Dual task performance improves when tasks are presented to separate hemispheres. Journal of Clinical and Experimental Neuropsychology, 8, 486-502.

Lopez, M., Kosson, D. S., Weissman, D. H., \& Banich, M. T. (2007). Interhemispheric integration in psychopathic offenders. Neuropsychology, 21, 82-93.

Luck, S. J., Hillyard, S. A., Mangun, G. R., \& Gazzaniga, M. S. (1989). Independent attentional systems mediate visual search in split-brain patients. Nature, 342, 543-545.

Merola, J. L., \& Liederman, J. (1985). Developmental changes in hemispheric independence. Child Development, 56, 1184-1195.

Merola, J. L., \& Liederman, J. (1990). The effect of task difficulty upon the extent to which performance benefits from between-hemisphere division of inputs. International Journal of Neuroscience, 51, 35-44.

Meyer, D. E., \& Kieras, D. E. (1997a). A computational theory of executive cognitive processes and multiple-task performance: Part 1. Basic mechanisms. Psychological Review, 104, 3-65.

Meyer, D. E., \& Kieras, D. E. (1997b). A computational theory of executive cognitive processes and multiple-task performance: Part 2. Accounts of psychological refractory-period phenomena. Psychological Review, 104, 749-791.

Norman, W. D., Jeeves, M. A., Milne, A., \& Ludwig, T. (1992). Hemispheric interactions: The bilateral advantage and task difficulty. Cortex, 28, 623-642.

Passarotti, A., Banich, M. T., Sood, R. K., \& Wang, J. M. (2002). A generalized role of interhemispheric interaction under attentionally demanding conditions: Evidence from the auditory and tactile modality. Neuropsychologia, 40, 1082-1096.

Pollman, S., Zaidel, E., \& von Cramon, D. Y. (2003). The neural basis of the bilateral distribution advantage. Experimental Brain Research, 221, 322-333.

Reuter-Lorenz, P. A., Stanczak, L., \& Miller, A. C. (1999). Neural recruitment and cognitive aging: Two hemispheres are better than one, especially as you age. Psychological Science, 10, 494-500.

Ruthruff, E., Miller, J., \& Lachmann, T. (1995). Does mental rotation require central mechanisms? Journal of Experimental Psychology: $\mathrm{Hu}$ man Perception and Performance, 21, 552-570. 
Singh, H., \& O’Boyle, M. W. (2004). Interhemispheric interaction during global-local processing in mathematically-gifted adolescents, averageability youth, and college students. Neuropsychology, 18, 371-377.

Sohn, Y.-S., Liederman, J., \& Reinitz, M. T. (1996). Division of inputs between hemispheres eliminates illusory conjunctions. Neuropsychologia, 34, 1057-1068.

Springer, S. P., \& Deutsch, G. (1998). Left brain and right brain (5th ed.). New York: W. H. Freeman.

Townsend, J. T., \& Wenger, M. J. (2004). The serial-parallel dilemma: A case study in a linkage of theory and method. Psychonomic Bulletin \& Review, 11, 391-418.

Tsukiura, T., Fujii, T., Fukatsu, R., Otsuki, T., Okuda, J., Umetsu, A., et al. (2002). Neural basis of the retrieval of people's names: Evidence from brain-damaged patients and fMRI. Journal of Cognitive Neuroscience, 14, 922-937.

Van Selst, M., \& Jolicoeur, P. (1994). Can mental rotation occur before the dual-task bottleneck? Journal of Experimental Psychology: Human Perception and Performance, 20, 905-921.

Weissman, D. H., \& Banich, M. T. (1999). Global-local interference modulated by communication between the hemispheres. Journal of Experimental Psychology: General, 128, 283-308.
Weissman, D. H., \& Banich, M. T. (2000). The cerebral hemispheres cooperate to perform complex but not simple tasks. Neuropsychology, 14, 41-59.

Weissman, D. H., Banich, M. T., \& Puente, E. I. (2000). An unbalanced distribution of inputs across the hemispheres facilitates interhemispheric interaction. Journal of the International Neuropsychological Society, 6, 313-321.

Yoshizaki, K. (2000). Stages of functional processing and the bihemispheric recognition of Japanese Kana script. Laterality, 5, 155-166.

Yoshizaki, K., \& Tsuji, Y. (2000). Benefits of interhemispheric integration on the Japanese Kana script-matching tasks. Perceptual and Motor Skills, 90, 153-165.

Zacks, J., Rypma, B., Gabrieli, J. D., Tversky, B., \& Glover, G. H. (1999). Imagined transformations of bodies: An fMRI investigation. Neuropsychologia, 37, 1029-1040.

Zhang, W., \& Feng, L. (1999). Interhemispheric interaction affected by identification of Chinese characters. Brain and Cognition, 39, 93-99.

Received October 19, 2005

Revision received October 23, 2006

Accepted October 30, 2006

\section{American Psychological Association SUBSCRIPTION ClaIMS INFORMATION}

Today's Date:

We provide this form to assist members, institutions, and nonmember individuals with any subscription problems. With the appropriate information we can begin a resolution. If you use the services of an agent, please do NOT duplicate claims through them and directly to us. PLEASE PRINT CLEARLY AND IN INK IF POSSIBLE.

\begin{tabular}{llll}
\hline PRINT FULL NAME OR KEY NAME OF INSTITUTION & & \\
\hline ADDRESS & & & \\
\hline CITY & & & \\
\hline
\end{tabular}

YOUR NAME AND PHONE NUMBER

\section{MEMBER OR CUSTOMER NUMBER (MAY BEFOUND ON ANY PAST ISSUE LABEL)} DATE YOUR ORDER WAS MAILED (OR PHONED)

_ PREPAID CHECK $\frac{\text { CHARGE }}{\text { CHECK/CARD CLEARED DATE: }}$

(If possible, send a copy, front and back, of your cancelled check to help us in our research of your claim.)

ISSUES: DAMAGED

TITLE

VOLUME OR YEAR

NUMBER OR MONTH

Thank you. Once a claim is received and resolved, delivery of replacement issues routinely takes 4-6 weeks.

(TO BE FILLED OUT BY APA STAFF)

DATE RECEIVED:

ACTION TAKEN:

STAFF NAME:
DATE OF ACTION:

INV. NO. \& DATE:

LABEL NO. \& DATE:

Send this form to APA Subscription Claims, 750 First Street, NE, Washington, DC 20002-4242

PLEASE DO NOT REMOVE. A PHOTOCOPY MAY BE USED. 